When the catgut is in position the loop between the two eyes should be pulled out several inches before withdrawing the needle. Otherwise withdrawal is difficult, and the catgut may become frayed. Fig. 3 shows one suture in position and the needle being threaded for the introduction of a second.

The twin needles are made in several sizes, and with varying spaces between the two. They are supplied by Messrs. Down Bros., London.

\section{Thimble Guide}

A thimble on the first finger acts as a guide on which a quick opening may be made for counterdrainage in cases of septic peritonitis (Fig. 4). The model now recommended is made by Weiss. It should fit the finger tightly. When the hand is passed from the main incision within the abdomen towards the suprapubic region the thimble is kept closely applied to the deep aspect of the abdominal wall, so that there is no possibility of a loop of small intestine being pushed forward and injured by the knife. A small incision is rapidly made on the finger guarded by the thimble; a tube is slipped over the top of the thimble and guided to the bottom of the pelvis by the hand. The illustration depicts a glass tube; a rubber variety is now more commonly employed. The thimble is of use in many situations (such as the axilla after breast amputation) where counterdrainage is needed.

\section{PERFORATED TYPHOID ULCER}

\section{Notes on Three Successful Cases}

BY

\section{J. B. G. MUIR, M.B., B.S., F.R.C.S., AND}

S. C. CHIA, M.B., B.S.

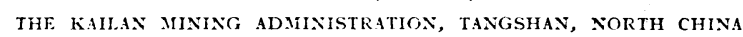

Perforation of typhoid ulcer occurs as a complication in about 3 per cent. of all cases of the disease, and, according to various authorities, accounts for approximately 30 per cent. of the total mortality. There is but one form of treatment-namely, operation as soon as possible. This is attended by a very high death rate. Harte and Ashhurst, ${ }^{1}$ in their classical paper on this subject, based on a large series of cases, quote it as 74 per cent., with decided increases depending upon the twelve-hour periods elapsing between the occurrence and surgical intervention. The rate is distinctly lower in children, being given as 50 per cent. by Jobson and Gitting, ${ }^{2}$ and as 36 per cent. by Elsberg. ${ }^{3}$

\section{Causes of High Mortality}

The factors responsible for this high mortality may be summarized as follows:

1. The catastrophe occurs generally during the second to third week of the disease, when the patient's general condition is at its worst, with severe toxaemia and a weak and dilated heart as contraindications to the necessary shock attending surgical intervention.

2. Occasionally it is very difficult to make a diagnosis of actual perforation, particularly in cases where great abdominal discomfort, pain, and distension have been dominating symptoms from the onset of the disease. (In such cases, however, a leucocytosis will always indicate the onset of peritonitis, and in any event it is probably better to submit even a weak and debilitated patient to the risk of laparotomy than to overlook a perforation until it is too late.)

3. The perforation may be complicated and temporarily masked by the simultaneous occurrence of intestinal haemorrhage from the separation of sloughs.

4. As a general rule, in cases of perforated typhoid ulcer, infection of the general peritoneal cavity by the extravasated intestinal contents is present from the first, with the formation of a watery exudate, and with little attempt at localization, or the formation of adhesions. (This was a feature of Cases I and II, described below.)

5. Difficulty is frequently experienced in suturing the perforation owing to the inflamed and friable condition of the bowel caused by adjacent ulceration. This has led some surgeons to treat the condition by passing an enterostomy tube through the perforation, fixing it in situ by a catgut stitch, and contenting themselves by draining the affected loop of bowel. Two successful cases treated in this way have recently been published by Branch. ${ }^{4}$

6. Occasionally perforations may be multiple, two, or even three, being present, and the necessary handling of the intestine during the operation may cause the perforation of adjacent ulcers.

7. Leakage frequently occurs, after apparently successful suture, resulting in the formation of a faecal fistula. These possibilities (Nos. 5, 6, and 7) have caused Roux-Berger, ${ }^{5}$ Cadenat, ${ }^{6}$ and others to treat the perforated loop of bowel by exteriorization, in addition to suture or enterostomy, but this method does not appear to have lowered the mortality.

8. Pulmonary complications, notably pneumonia, are common sequels of the operation.

\section{The High Death Rate in China}

In China the mortality tends to be still higher, as, in addition to the above considerations, there is the fact that the majority of patients wait until their conditions are literally hopeless before submitting to operation. An interesting point in the local occurrence of the disease has been observed by Loucks, ${ }^{7}$ who considers that in some years typhoid infections show a much greater virulence than in others, and that there is then a much higher incidence of perforation. In view of these facts, the first and second cases here described are encouraging in that twenty and forty hours elapsed respectively between perforation and operation, which was successful, and the third case is interesting as illustrating how localization of a typhoid perforation in a hernial sac may simulate a strangulated inguinal hernia.

\section{CASE I (J. B. G. M.)}

Perforation of typhoid ulcer, with operation twenty hours after onset: recovery.

L. W., aged 11, daughter of a Chinese merchant, was suffering from a severe attack of typhoid fever (Widal ++ ). On September 15th, during the third week of the discase, she was suddenly seized with acute pain in the lower abdomen, with vomiting, and became very collapsed. Hes medical attendant, Dr. T. J. Hua, saw her half an hour later, when abdominal distension and rigidity were becoming apparent, and diagnosed perforation of a typhoid ulcer, a diagnosis which was subsequently confirmed by two colleagues. Operation was advised, and after considerable discussion consent of the family was obtained; I was eighty-five miles away when telegraphed for. Unfortunately, owing to intrequent trains, I was unable to arrive before 6.30 the following morning. During this time the patient was kejt in the Fowler position, continuous " drip" rectal saline cont:ining 5 per cent. glucose was administered, and 5 minims of tincture of opium was given by the mouth four-hourly. I performed laparotomy, under open ether anaesthesia, at 7 a.m. on September 16th, twenty hours after the perforation had occurred. A large quantity of seropurulent fluid escaped from the abdomen when the peritoneum was opened, but there was surprisingly little congestion or lymphatic exudation on the intestinal coils. A small circular perforation in the base of an ulcer was found, situated on the free border of the ileum, about $\mathbf{8}$ inches from the caecum, and was closed by invaginating purse-string sutures of chromic catgut. The extravasated fluid was thoroughly mopped up, and the abdomen was closed with a large pelvic drain in situ, the whole proceeding occupying only eight minutes. The chill suffered from severe shock, and her condition was very guve for about twenty-four hours, when she began to rally, and made 
a good recovery. Her convalescence, though protracted, was very satisfactory, despite a small faecal fistula which persisted at the site of the pelvic drainage tube for about a month before closing spontaneously.

\section{CASE II (S. C. C.)}

Perforation of typhoid ulcers complicating pregnancy, with operation forty hours after onset: recovery.

L. H. A., aged 28 , wife of a Chinese military officer, was admitted to hospital on November 18th, 1930, with the following history. She had been ill for twelve days, suffering from fever, headache, and abdominal discomfort. Following the ingestion of a herbal remedy reconmended by a native " nedicine man" she was seized with acute abdominal pain on the evening of November 16th. She vomited several times at the onset of the attack, and became very collapsed. The native practitioner treated her with morphine injections, but as her condition was rapidly becoming worse her relatives brought her to hospital at $6 \mathrm{p} . \mathrm{m}$. on November 18th, forty hours after the onset of the acute pain, and, presumably, of the occurrence of perforation. The case was complicated by the fact that the patient was five months pregnant, but as it was obvious that peritonitis was present and that the patient was in extremis, I performed immediate laparotomy. On opening the peritoneum a quantity of gas escaped, with a lot of thin seropurulent fluid. The pregnant uterus filled the cntire pelvis, and the inflamed and congested intestine, with considerable lymphatic exudation on the surface, appeared to occupy the entire abdomen. Two perforations were found on the free border of a coil of the ileum, about 12 inches from the caecum. They were about 2 inches apart; and were each closed with some difficulty, owing to the extreme friability of the bowel, by purse-string catgut sutures. Thorough irrigation of the entire peritoneal cavity was then carried out, using several pints of warm normal saline, and the abdomen was closed, three large tubes being left in situ, draining both flanks and the mid-line. The patient's condition was exceedingly grave for two days following operation, severe abdominal distension being present, but turpentine encmas caused the bowels to act on the second day, with considerable relief of symptoms. Matters were further complicated by the occurrence of a miscarriage on the third day following operation, a five-month foetus being expelled. The patient's condition however, did not seem to be greatly impaired by this mishap. On the eighth day following operation a severe attack of broncho-pneumonia developed, and for several days it was thought that this would prove a fatal complication. After several weeks' illness, however, the patient slowly rccovered, and was discharged in good health from hospital eleven weeks alter admission.

Consideration of these two histories makes it clear that it is never too late to operate in a case of perforated typhoid ulcer, as even the apparently hopeless patients (vide Case II) may possess amazing recuperative powers. An interesting and common feature to both cases was the thin, watery character of the peritoneal exudate, with flakes of yellowish-white lymph-not nearly so offensive as the exudate in many types of appendicular peritonitis. Unfortunately in Case I no bacteriological examination was made, but in Case II smear and culture showed staphylococci and Gram-negative bacilli.

Harte and Ashburst, ${ }^{1}$ in considering the bacteriology, have pointed out that the prognosis is graver if the streptococcus is the predominating organism, as, in cases in which the typhoid bacillus alone is the infecting cause, the peritonitis is of a relatively low-grade type, with a watery exudation and few adhesions. It is probable, therefore, that the successful results in these two cases were largely influenced by this factor.

\section{CASE III (J. B. G. M.)}

A Chinese casual labourer, aged 32, suffered since boyhood from a right inguinal hernia, which had latterly increased considerably in size, but, as he somewhat graphically put it, he could push his bowels back into his stomach when he lay down. On October 2nd, 1930, he was admitted to the Tongshan
Base Hospital as a case of strangulated inguinal hernia. The history was that for the preceding fortnight he had been feeling unwell, suffering from headache, some diarrhoea, fever, and swelling of his stomach. Several days before intmission he had experienced a good deal of pain in his hernial swelling, and found that it could not be reduced. He used considerable force in attempting reduction on the day of admission, and the pain became exceedingly severe. The inguinal swelling was very tense, with the skin over it very red, and the patient's condition simulated intestinal obstruction due to strangulation of the inguinal hernia. Immediate operation was advised and agreed to. On incising the sac much purulent fluid escaped, and a coil of ileum, about 18 inches in length, was seen to be incarcerated. It was very swollen and congested, but obstructed rather than strangulated. A small round perforation was present on the free border of the coil, through which the extravasation of intestinal contents had occurred, and several ulcers could be palpated along the free border of the bowel. The perforation was closed by an invaginated purse-string suture, the intestinal loop carefully washed with warm saline solution, and the loop of bowel returned to just within the abdominal cavity. A large drainage tube was inserted through the neck of the sac down to the sutured perforation, and the patient returned to bed. A subsequent blood examination gave a strongly positive Widal reaction, and the later course of the case was that of typical typhoid fever. A radical cure was performed for the hernia two months later.

In this case the hernia was undoubtedly rendered irreducible by the inflammatory swelling and infiltration of the Peyer's patches in the herniated bowel, as a consequence of typhoid. It was indeed a lucky coincidence that the perforation occurred in the hernial sac rather than in the general peritoneal cavity, although it is quite probable that perforation was induced by the patient's own attempts at reduction of the inflamed bowel.

\section{ReFerences}

1 Harte and Ashhurst: Annals of Surgery, 1904, xxxix.

2 Jobson and Gitting: Amer. Journ. of Med. Science, November, 1909

3 Elsberg: Annals of Surgery, July, 1903.

1 Branch.J. R. B.: Ibid., March, 1928

5 Rolux-Berger, J. L.: Bulletin et Mémoires de la Société Nationalo (Paris), February, 1930, lvi, et discussion.

- Cadenat: Ibid.

${ }^{7}$ Loucks, H. N.: Personal communication, from Department of Surgery, Peiping Union Medical College, Peiping.

\section{TUBERCULOUS PERITONITIS SIMULATING COELIAC DISEASE}

\section{By}

\section{E. C. WARNER, M.D., M.R.C.P.}

PHYSICIAN TO THE CHILDREX'S DEPARTMENT, THE MILLER GFNERAL HOSPITAL ; PHYSICLN WITH CHARGE OF OUT-PATILNTS, THE DREADNUCGHI HOSPITAL, GREENWICH

In view of the interest aroused in coeliac disease by the excellent review of Leonard Parsons, ${ }^{1}$ and by the theory put forward by $\mathrm{Ryle}^{2}$ that the essential cause is an obstruction of the lacteals by previous inflammation, it seems opportune to record a case recently under my care in the Miller General Hospital, which closely mimicked coeliac disease, and in which extensive tuberculous peritonitis was found at necropsy.

\section{CASE History}

Mary O. was first referred to me by Dr. Rogerson of Bexley Heath, on account of diarrhoea, abdominal enlarge. ment, and wasting. In this family the seven other children were healthy. At birth she was a full-term baby weighing $6 \mathrm{lb}$., and she was breast-fed. She remained well till two months before I first saw her, when, at the age of 2 years and 4 months, she began to get periodic attacks of diarrhoea with occasional pale stools, associated with loss of weight, feverish turns, whining, abdominal enlargement, and occasionally prolapse of the rectum. At no time was there any. 